\title{
Survey on Neural Network Architectures with Deep Learning
}

\author{
Dr. S. Smys, \\ Professor, \\ Department of CSE, \\ RVS Technical Campus, \\ Coimbatore, India. \\ Email id:smys375@gmail.com \\ Dr. Joy Iong Zong Chen, \\ Professor, Department of Electrical Engineering, \\ Da-Yeh University, \\ Taiwan. \\ Email id: jchen@mail.dyu.edu.tw

\section{Dr. Subarna Shakya,} \\ Professor, Department of Electronics and Computer Engineering, \\ Central Campus, Institute of Engineering, Pulchowk, \\ Tribhuvan University, \\ Pulchowk, Lalitpur, Nepal. \\ Email:drss@ioe.edu.np.
}

\begin{abstract}
In the present research era, machine learning is an important and unavoidable zone where it provides better solutions to various domains. In particular deep learning is one of the cost efficient, effective supervised learning model, which can be applied to various complicated issues. Since deep learning has various illustrative features and it doesn't depend on any limited learning methods which helps to obtain better solutions. As deep learning has significant performance and advancements it is widely used in various applications like image classification, face recognition, visual recognition, language processing, speech recognition, object detection and various science, business analysis, etc., This survey work mainly provides an insight about deep learning through an intensive analysis of deep learning architectures and its characteristics along with its limitations. Also, this research work analyses recent trends in deep learning through various literatures to explore the present evolution in deep learning models.
\end{abstract}

Keywords: - Deep Learning, Architectures, Algorithms

\section{Introduction}

Machine learning is a popular approach in present research environment as it deals various applications in image processing, signal processing and other data analysis [1]. In particular, deep learning gains more importance due to its immense characteristics in image classification [2], data mining, language and speech processing applications [3]. The remarkable growth of data and development in technologies have brought various key routes in deep learning models and makes the system to outperforms better than earlier learning models. Conventional machine learning models perform tasks based on the features extracted from the input data while deep learning uses graph technologies along with neuron transformations to obtain multilayer learning models and learns the data automatically without any difficulty. The most important deep learning model which is widely used is artificial neural network (ANN), but there are many. Major difference between the conventional machine learning model and deep learning model is its automatic learning process which makes deep learning suitable for wide range of applications. Some of the major deep learning models are

- Autoencoders - It is an artificial neural network which has the ability to learn various coding patterns. In this the output layer has same number of nodes as input layer and it predicts the inputs instead of predicting the output vectors.

- Deep Belief Network - It is suitable for handling nonconvex objective functions while using multilayer perception. 
- Convolutional Neural Network $(\mathrm{CNN})$ - It is type of feedforward neural network where the individual neurons are arranged to respond to all regions in the area.

- Recurrent Neural Networks (RNN) - It allows to operate over sequence of vectors in input and output as a directed cycle and shares its parameters at every layer so that it could be trained depends on the application.

- $\quad$ Reinforcement learning - It is a hybrid programming supervised learning model.

\section{Deep Learning Architectures}

Based on the applications and types of neural networks, deep learning architecture is classified into three major classes. Figure 1 gives an illustration of architecture types in deep learning.

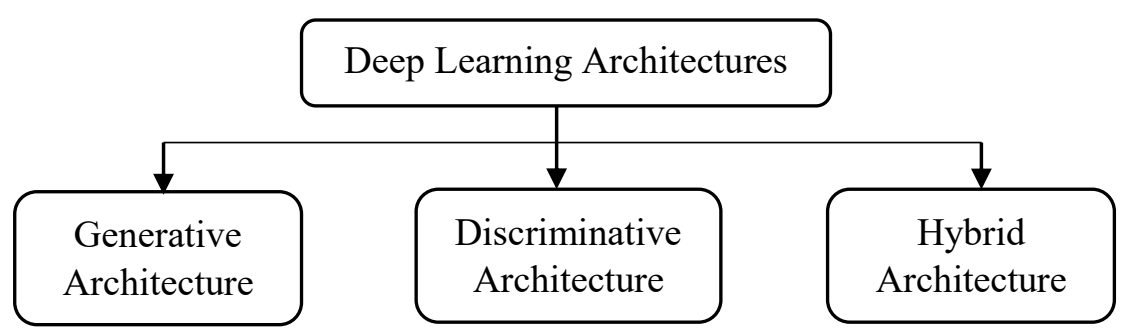

\section{i) Generative Architecture}

Fig.1 Types of Deep Learning Architecture

Generative architecture based deep learning models are generally termed as unsupervised feature learning models as the data labels are not considered in this approach. Supervised learning and unsupervised pretraining is the key concept in generative deep learning architectures. This type of architecture is evolved when there is limited data to train for the difficult network, these models learns the lower level of data and provides necessary solutions without depends on the other layers.

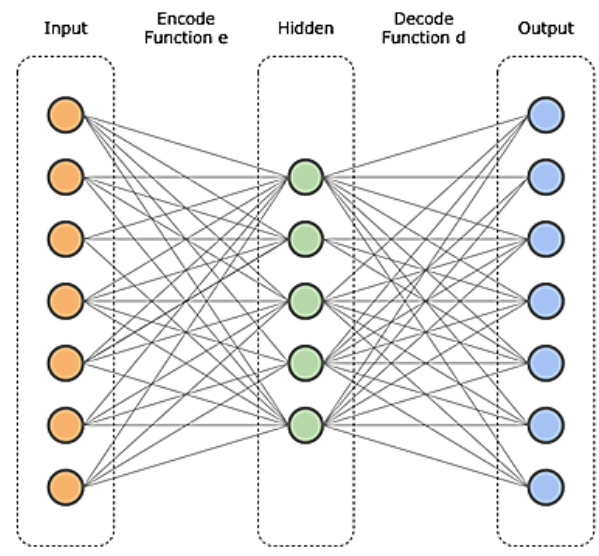

Fig.2 Deep Autoencoder

Autoencoder is one of the familiar generative architecture models in which a vector is considered as input and the network tries to match the output function to the vector by reframing the input dimensions. This helps to construct low or high dimensional data so that autoencoder is widely used in various applications. Illustrative representation of deep autoencoder is depicted in figure 2 . Autoencoders learn the compressed data by coding it in a supervised manner and train single layer for each process which reduces the computation resources. Network is used to encode the data if the input and output layer has higher dimensionality than hidden layer which is termed as feature compression in autoencoder process. In some cases, it acts a s denoising autoencoders which reconstructs the input signal from a noisy input signal. Stacked autoencoder and sparse autoencoder are some other versions in autoencoder where two or more hidden nodes are used to activate the function. 
Deep Boltzmann machine is another generative architecture model in which many hidden layers are used and there is no physical connection between the variables in the same layer. It is designed based on the conventional Boltzmann machine which is used to obtain decisions through a symmetrical network which has nodes are connected symmetrically. Conventional learning models are complex to analyse and performs less while in learning, deep Boltzmann machine is developed. In this each layer is used to obtain the high order correlation coefficients between the hidden features and the below layer. Due to this this machine has the ability to learn internal complex representations and provides solutions for signal processing applications. If the hidden layers in deep Boltzmann machine is reduced into one, then it is called as restricted Boltzmann machine. Through this restricted machine various hidden layers could be effectively learned through its activation features of a single machine which is used to train the next layer. Conventional deep Boltzmann machine is improved as high order Boltzmann machine in the bottom layer as mean covariance restricted Boltzmann machine reduces the limitations in conventional models. Though it has various advantages, the important issues in deep Boltzmann machines are its training difficulty and high-level architecture. Figure 3 gives an illustration of restricted Boltzmann machine.

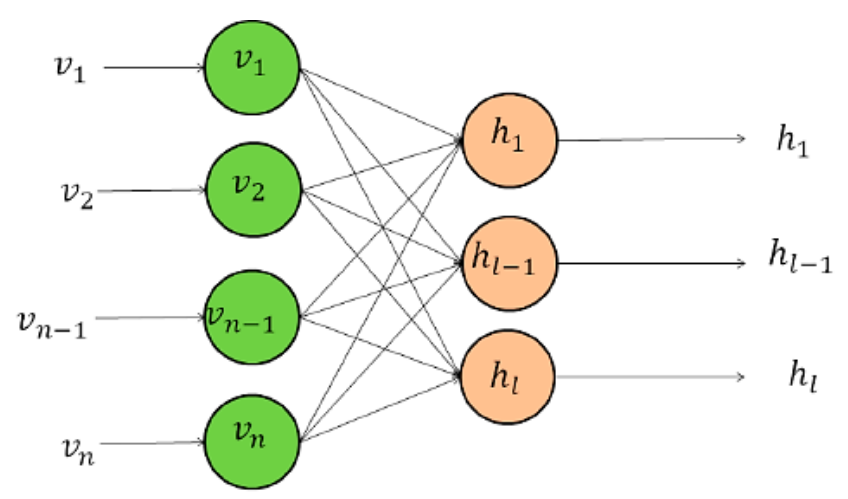

Fig.3 Restricted Boltzmann machine

Sum product network is another generative architecture type deep network which used directed acyclic graph as data leaves and the internal operations are performed in the nodes of the deep architecture. The sum nodes in the architecture provides mixture model and the product provides the features with definite hierarchy. The learning process in sum product is performed based on expectation maximization algorithm along with back propagation. The learning process starts from finding the structure of the network from its weights. The issues in this network is its learning signal which quickly dilutes when it is propagated in the deep layers. The difficulty in weights and its discriminative information the effectiveness of the system reduces in classification tasks and this could be overcome through backpropagation training algorithm which was introduced later. This algorithm utilizes the gradient descent and computes the derivatives along with the conditions to reduce the diffusion problem in deep networks.

Recurrent neural network is one of the important generative architecture type deep learning model which is used to generate and analyse the sequential data. Based on the input data length, the depth of the neural network will vary and it is an important neural network model used in speech or data processing applications. The general issue in recurrent neural network is its gradient vanishing issue. Due to this training is extremely difficult in RNN so that it is applied into very limited research areas. These gradient issues are reduced in the recent times due to the development of optimization models. various research works are evolved based on RNN, in which language modelling, text recognition are some of the important research models. Figure 4 gives an illustration of RNN.

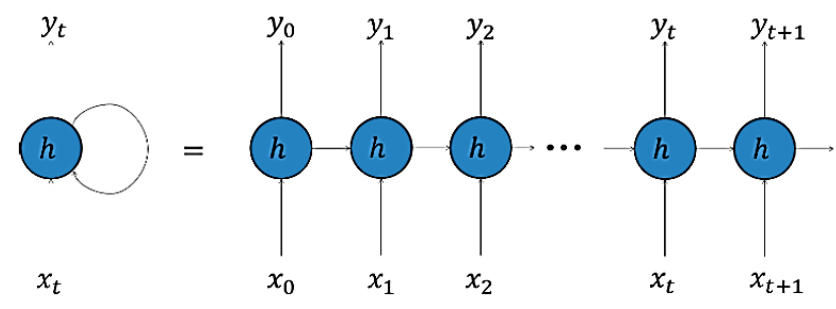

Fig.4 RNN- Recurrent Neural Network

ISSN: 2582-2640 (online) 
Recursive deep generative models are used to obtain human modelling for natural scene parsing and natural language processing. This learning algorithms helps to determine the model structure with minimum learning parameters compared to other architecture models. It is used to process images and language sentences through max-margin prediction architecture. It helps to analyse the sentences or images and interact with each unit to provide a complete sustained output. Table 1 provides a summary analysis of generative architectures under various domains.

Table 1 Analysis of generative architecture models

\begin{tabular}{|c|c|c|c|c|}
\hline Methods & $\begin{array}{l}\text { Reference } \\
\text { article }\end{array}$ & Application & Merits & Demerits \\
\hline \multirow{7}{*}{ Autoencoder } & [4] & $\begin{array}{l}\text { Facial Expression } \\
\text { Recognition }\end{array}$ & $\begin{array}{l}\text { Improved } \\
\text { efficiency than } \\
\text { conventional } \\
\text { models }\end{array}$ & $\begin{array}{l}\text { Lags in recognition } \\
\text { performance over } \\
\text { reduced dimensional } \\
\text { data }\end{array}$ \\
\hline & {$[5]$} & Image Denoising & $\begin{array}{l}\text { Better Image } \\
\text { enhancement }\end{array}$ & $\begin{array}{l}\text { Not suitable for wide } \\
\text { range of applications }\end{array}$ \\
\hline & {$[6]$} & pattern recognition & $\begin{array}{l}\text { Efficient matching } \\
\text { performance }\end{array}$ & $\begin{array}{l}\text { Lags for complex } \\
\text { patterns and time } \\
\text { consuming }\end{array}$ \\
\hline & {$[7]$} & Speech processing & $\begin{array}{l}\text { Improved } \\
\text { performance }\end{array}$ & System complexity \\
\hline & [8] [9] & Fault Diagnosis & $\begin{array}{l}\text { Efficient fault } \\
\text { detection }\end{array}$ & $\begin{array}{l}\text { System shows invariant } \\
\text { performance for } \\
\text { different types of faults. }\end{array}$ \\
\hline & {$[10][11]$} & Medical Data Analysis & $\begin{array}{l}\text { Reliable and cost } \\
\text { efficient }\end{array}$ & Complex design \\
\hline & {$[12]$} & Anomaly detection & $\begin{array}{l}\text { Efficiency and } \\
\text { robustness }\end{array}$ & Implementation Cost \\
\hline \multirow{4}{*}{$\begin{array}{l}\text { Deep } \\
\text { Boltzmann } \\
\text { Machines }\end{array}$} & [13] & Feature selection & High Efficiency & Needs external support \\
\hline & {$[14]$} & Industrial fault diagnosis & $\begin{array}{l}\text { Efficient fault } \\
\text { detection }\end{array}$ & $\begin{array}{l}\text { Lags in detection } \\
\text { accuracy for minor } \\
\text { faults }\end{array}$ \\
\hline & {$[15]$} & Medical Image processing & $\begin{array}{l}\text { High Prediction } \\
\text { accuracy }\end{array}$ & Computation Cost \\
\hline & [16] & $\begin{array}{l}\text { Bio Medical image } \\
\text { processing }\end{array}$ & High Efficiency & $\begin{array}{l}\text { System complexity and } \\
\text { computation cost }\end{array}$ \\
\hline $\begin{array}{l}\text { Restricted } \\
\text { Boltzmann } \\
\text { Machines }\end{array}$ & $\begin{array}{l}{[17][18]} \\
{[19]}\end{array}$ & $\begin{array}{l}\text { Time series forecasting, } \\
\text { gradient approximation, } \\
\text { input weight } \\
\text { determination }\end{array}$ & $\begin{array}{l}\text { Better performance } \\
\text { in gradient issues }\end{array}$ & $\begin{array}{l}\text { Restricted approach } \\
\text { which is suitable for few } \\
\text { applications }\end{array}$ \\
\hline $\begin{array}{l}\text { sum-product } \\
\text { network }\end{array}$ & {$[20][21]$} & $\begin{array}{l}\text { scene detection, image } \\
\text { processing, acoustic } \\
\text { relation }\end{array}$ & $\begin{array}{l}\text { Simple and } \\
\text { efficient }\end{array}$ & $\begin{array}{l}\text { Not suitable for wide } \\
\text { range of applications }\end{array}$ \\
\hline \multirow{5}{*}{$\begin{array}{c}\text { Recurrent } \\
\text { Neural Network }\end{array}$} & {$[22]$} & Document image analysis & $\begin{array}{l}\text { Effective } \\
\text { document } \\
\text { management }\end{array}$ & $\begin{array}{l}\text { Requires external } \\
\text { support and lags over } \\
\text { large dimensional data }\end{array}$ \\
\hline & {$[23][24]$} & Handwriting Recognition & $\begin{array}{l}\text { Better recognition } \\
\text { performance }\end{array}$ & $\begin{array}{l}\text { Lags in detecting mixed } \\
\text { words }\end{array}$ \\
\hline & {$[25]$} & $\begin{array}{l}\text { Biomedical Image } \\
\text { processing }\end{array}$ & $\begin{array}{l}\text { Better detection } \\
\text { rate }\end{array}$ & $\begin{array}{l}\text { High computation cost } \\
\text { and time }\end{array}$ \\
\hline & {$[26]$} & software engineering & Automated process & $\begin{array}{l}\text { Limited to very specific } \\
\text { applications }\end{array}$ \\
\hline & {$[27]$} & channel estimation & $\begin{array}{l}\text { Better estimation } \\
\text { performance }\end{array}$ & $\begin{array}{l}\text { Lags over noise and } \\
\text { other interferences }\end{array}$ \\
\hline
\end{tabular}




\section{ii)}

\section{Discriminative Architectures}

Discriminative architectures are widely used in information and signal processing as a shallow architecture along with Hidden Markov Model or Conditional random fields. Recently deep structures with conditional random fields are evolved, in which the output of each lower layer of random field is stacked with original input data which is on higher layer. Discriminative architectures are widely used in language processing and recognition applications. Various research works are evolved under discriminative architecture, in this backpropagation learning based speech recognition is one of the important researches. Based on the emission probabilities in hidden Markov models, a discriminative learning neural network model tandem is evolved. In this the features of HMM are observed based on the activities of hidden layers as various combinations which forms a discriminative architecture. Deep stacking networks is another recent development under discriminative architecture based on tensor variant to solve the discriminations through learning on scalable generative components. In some research works RNN is used as discriminative architectures. In general, RNN is used to generate output based on the prediction of input data. If the output is associated with the input data then it could be used as a discriminative model. In order to train RNN as discriminative model, the training data are need to be pre-segmented also it requires a post processing process to convert the outputs into desired data sequences. This is the limitation in RNN when it used as discriminative models, since the segmentation and training cost increases the system overall cost.

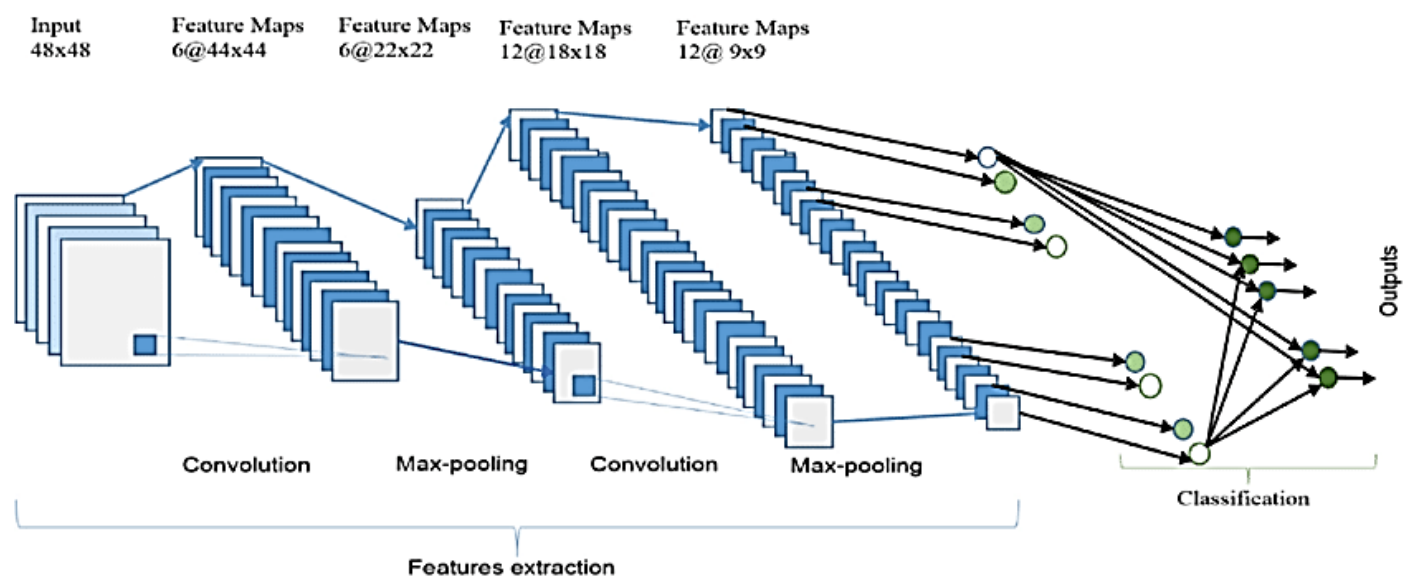

Fig.5 Convolution Neural Network

Convolutional neural network (CNN) is one of the important discriminative architecture. CNN consists of a pooling layer and convolution layer in its architecture. To form a deep model these two modules are stacked one over the other. The convolution layer is used to share the weights and pooling layer is used to subsample the convolution layer output. This process greatly reduces the data rate below the data rate in other neural network models. CNN has its invariance property due to its convolution layer weight sharing process and its unique pooling schemes. CNN is widely used in various applications in image and signal processing. Figure 5 gives an illustration of CNN model. Table 2 describes the applications of discriminative architectures in various domains with its merits and demerits.

Table 2. Analysis Summary of Discriminative architectures

\begin{tabular}{|c|c|c|l|l|}
\hline Methods & $\begin{array}{c}\text { Reference } \\
\text { article }\end{array}$ & \multicolumn{1}{|c|}{ Application } & \multicolumn{1}{|c|}{ Merits } & \multicolumn{1}{c|}{ Demerits } \\
\hline $\begin{array}{c}\text { Adaptive } \\
\text { discriminative } \\
\text { Learning }\end{array}$ & {$[28]$} & $\begin{array}{c}\text { Scene } \\
\text { Recognition }\end{array}$ & $\begin{array}{l}\text { Better Recognition } \\
\text { efficiency }\end{array}$ & Slow Learning Rate \\
\cline { 2 - 5 } & {$[29]$} & Image Retrieval & Computation time & $\begin{array}{l}\text { Less efficient compared } \\
\text { to other neural network } \\
\text { models }\end{array}$ \\
\hline
\end{tabular}




\begin{tabular}{|c|c|c|c|c|}
\hline & {$[30][31]$} & Face Recognition & $\begin{array}{l}\text { Improved recognition } \\
\text { than conventional } \\
\text { learning models }\end{array}$ & computation cost \\
\hline & {$[32]$} & $\begin{array}{l}\text { Real time image } \\
\text { analysis }\end{array}$ & $\begin{array}{l}\text { Better classification } \\
\text { performance }\end{array}$ & High processing time \\
\hline & {$[33]$} & $\begin{array}{c}\text { Data } \\
\text { classification }\end{array}$ & $\begin{array}{l}\text { high classification } \\
\text { efficiency }\end{array}$ & $\begin{array}{l}\text { high computational } \\
\text { overhead }\end{array}$ \\
\hline & {$[34][35]$} & $\begin{array}{c}\text { Person } \\
\text { Identification }\end{array}$ & Improved accuracy & $\begin{array}{l}\text { accuracy could be } \\
\text { improved further }\end{array}$ \\
\hline & {$[36]$} & Image processing & $\begin{array}{l}\text { better performance in } \\
\text { image blur detection }\end{array}$ & $\begin{array}{l}\text { less efficient for low } \\
\text { dimensional images }\end{array}$ \\
\hline \multirow{6}{*}{$\begin{array}{l}\text { Convolution } \\
\text { Neural Network }\end{array}$} & {$[37]$} & Data Analysis & $\begin{array}{l}\text { Better classification } \\
\text { performance }\end{array}$ & $\begin{array}{l}\text { Lags in dimensionality } \\
\text { issues. }\end{array}$ \\
\hline & {$[38]$} & Lip reading & Efficient conversion & $\begin{array}{l}\text { Limitations in detecting } \\
\text { similar words }\end{array}$ \\
\hline & {$[39]$} & $\begin{array}{c}\text { Facial } \\
\text { Expression } \\
\text { Recognition }\end{array}$ & $\begin{array}{l}\text { Effective emotion } \\
\text { analysis and better } \\
\text { classification } \\
\text { performance }\end{array}$ & $\begin{array}{l}\text { Issues in analysing } \\
\text { similar expressions }\end{array}$ \\
\hline & {$[40]$} & $\begin{array}{c}\text { Posture } \\
\text { Recognition }\end{array}$ & $\begin{array}{l}\text { Suitable for wide range } \\
\text { of static applications }\end{array}$ & $\begin{array}{l}\text { Lags in analysis of } \\
\text { dynamic postures }\end{array}$ \\
\hline & {$[41]$} & $\begin{array}{c}\text { Biomedical } \\
\text { image processing }\end{array}$ & $\begin{array}{l}\text { Better classification } \\
\text { efficiency }\end{array}$ & System complexity \\
\hline & {$[42]$} & Data Mining & $\begin{array}{l}\text { Efficient data } \\
\text { classification and } \\
\text { management }\end{array}$ & $\begin{array}{l}\text { computation overhead } \\
\text { and cost }\end{array}$ \\
\hline
\end{tabular}

Hybrid architectures comprises of both generative and discriminative process [43]. In most of the hybrid architectures, the generative components are used along with discriminative components to attain the final solution. Since the generative models are used to solve nonlinear parametric issues which reduces the initialization issues. Also, generative models have regularized control features which reduces the complexity of the system. Deep neural network (DNN) is a prominent hybrid architecture [44] where the generative architecture of deep belief network is modified using discriminative architecture in training process deep neural network is evolved. In this the weights of deep neural network are pretrained using reduced Boltzmann machine for random initialization. In some research works deep belief network is used as an initialization factor for deep neural networks. In this case sequence level tuning is performed instead of frame level tuning.

Few research models use random fields along with deep neural network using the condition probability of labels and its input data sequence. This equivalent architecture is similar to architecture model of deep neural network with hidden Markov Model where the parameters are used to learn [45] the entire sequence with maximum information between the input and output vectors. Similar method is processed in shallow neural network and its uses discriminative training with minimum error technique. In few researches Restricted Boltzmann model is used to learn through discriminative architecture probabilities in which the label vectors are connected with the data vector which creates an overall layer of Restricted Boltzmann model. This improves the performance of Restricted Boltzmann model in classification issues. Deep belief network [46] is combined with random fields to learn the lower level features and recognize the images as a classification and recognition model. These generative models improve the performance of deep belief network. Deep convolutional neural networks are introduced as a hybrid architecture by combining CNN with deep belief networks. The discrimination in convolution neural network is its random initialization and it could be improved by incorporating deep belief network. This hybrid system is suitable for speech recognition and text recognition.

\section{Applications using machine learning}

In the above section based on three architectural level various application of deep learning models are analysed along with its merits and demerits. The application of deep learning is not limited to these categories and 
also extends in various domains like human activity recognition [47], social media sentiment analysis [48], smart activity monitoring [49], image and computer vision engineering [50], various medical applications [51], dropout prediction [52], agricultural applications [53], etc., Still there are many ongoing researches to improve the performance of system through machine learning approaches.

\section{Findings from the survey}

Based on the above intense survey the following findings are summarized as follows

- Generative models are suitable for simple image and signal processing applications.

- Autoencoder is widely used in facial recognition, speech recognition-based approaches in most of the research works.

- Less number of research works are evolved in deep Boltzmann and restricted Boltzmann models due to its limitations in layer selection.

- $\quad$ Recurrent neural network is limited with gradient issues and it is used in less number of applications.

- In case discriminative models, convolution neural network is a familiar and widely used technique.

- $\mathrm{CNN}$ based applications are introduced in speech recognition, image processing, signal processing, data analysis etc.,

- Hybrid architectures are the recent trends where the generative and discriminative architectures are combined to form this hybrid structures.

- Deep neural network is widely used architecture to incorporate with general architectures for developing hybrid structures.

- Deep convolution neural network is the recent trend in deep learning models which is a hybrid architecture performs better in numerous applications.

\section{Conclusion}

This literature review provides an insight of deep learning neural networks. Based on the applications and the design methods, deep networks are analysed and categorized into generative architectures, discriminative architectures and hybrid architectures. Under these three categories, various deep learning models are analysed along with its recent application-based approaches. Based on the observations the findings of the survey are summarized which will help everyone in future to improve the research work through any of the models. In this research point of view, it is recommended to improve the convolution neural network or deep convolution neural network-based models which is suitable for wide range of applications. The future research work could be introducing hybrid architectures in convolution neural network for better performance improvement.

\section{References}

1. Alessio Micheli, Frank-Michael Schleif, Peter Tiňo (2013). Novel approaches in machine learning and computational intelligence. Neurocomputing. 112,1-3.

2. Iti Chaturvedi, Yew-Soon Ong, Rajesh Vellore Arumugam (2015). Deep transfer learning for classification of time-delayed Gaussian networks. Signal Processing. 110, 250-262.

3. Daniel Michelsanti, Zheng-Hua Tan, Sigurdur Sigurdsson, Jesper Jensen (2019). Deep-learning-based audiovisual speech enhancement in presence of Lombard effect. Speech Communication. 115,38-50.

4. Nianyin Zeng, Hong Zhang, Baoye Song, Weibo Liu, Abdullah M. Dobaie (2018). Facial expression recognition via learning deep sparse autoencoders. Neurocomputing, 273, 643-649

5. Komal Bajaj, Dushyant Kumar Singh, Mohd. Aquib Ansari (2020). Autoencoders Based Deep Learner for Image Denoising. Procedia Computer Science. 171, 1535-1541.

6. Jianbo Yu, Xiaoyun Zheng, Shijin Wang (2019). A deep autoencoder feature learning method for process pattern recognition. Journal of Process Control. 79, 1-15

7. Hung-Ping Liu, Yu Tsao, Chiou-Shann Fuh (2018). Bone-conducted speech enhancement using deep denoising autoencoder. Speech Communication. 104, 106-112

8. Haidong Shao, Hongkai Jiang, Huiwei Zhao, Fuan Wang (2017). A novel deep autoencoder feature learning method for rotating machinery fault diagnosis. Mechanical Systems and Signal Processing. 95, 187-204

9. Abdul Mujeeb, Wenting Dai, Marius Erdt, Alexei Sourin (2019). One class-based feature learning approach for defect detection using deep autoencoders. Advanced Engineering Informatics. 42, 1-15 
10. Kannadasan, Damodar Reddy Edla, Venkatanareshbabu Kuppili (2019). Type 2 diabetes data classification using stacked autoencoders in deep neural networks. Clinical Epidemiology and Global Health. 7(4),530-535

11. Ozal Yildirim, Ru San Tan, U. Rajendra Acharya (2018). An efficient compression of ECG signals using deep convolutional autoencoders. Cognitive Systems Research. 52, 198-211

12. Niklas Renström, Pramod Bangalore, Edmund Highcock (2020), System-wide anomaly detection in wind turbines using deep autoencoders, Renewable Energy, 157, 647-659.

13. Aboozar Taherkhani, Georgina Cosma, T. M McGinnity (2018). Deep-FS: A feature selection algorithm for Deep Boltzmann Machines. Neurocomputing. 322, 22-37.

14. Guangzheng Hu, Huifang Li, Yuanqing Xia, Lixuan Luo (2018). A deep Boltzmann machine and multigrained scanning forest ensemble collaborative method and its application to industrial fault diagnosis. Computers in Industry. 100, 287-296.

15. Yifeng Li, François Fauteux, Jinfeng Zou, André Nantel, Youlian Pan (2019). Personalized prediction of genes with tumor-causing somatic mutations based on multi-modal deep Boltzmann machine. Neurocomputing. 324, 51-62.

16. Jian Wu, Thomas R. Mazur, Su Ruan, Chunfeng Lian, Hua Li (2018). A deep Boltzmann machine-driven level set method for heart motion tracking using cine MRI images. Medical Image Analysis. 47, 68-80.

17. Takashi Kuremoto, Shinsuke Kimura, Kunikazu Kobayashi, Masanao Obayashi (2014). Time series forecasting using a deep belief network with restricted Boltzmann machines. Neurocomputing. 137, 47-56.

18. Huihui Shen, Hongwei Li (2019). A gradient approximation algorithm based weight momentum for restricted Boltzmann machine. Neurocomputing. 361,40-49.

19. Andre G. C. Pacheco, Renato A. Krohling, Carlos A. S. da Silva (2018). Restricted Boltzmann machine to determine the input weights for extreme learning machines. Expert Systems with Applications. 961, 77-85.

20. Zehuan Yuan, Hao Wang, Limin Wang, Tong Lu, Chew Lim Tan (2016). Modeling spatial layout for scene image understanding via a novel multiscale sum-product network. Expert Systems with Applications. 63,231240.

21. Frank Rudzicz, Arvid Frydenlund, Sean Robertson, Patricia Thaine (2016). Acoustic-articulatory relationships and inversion in sum-product and deep-belief networks. Speech Communication. 79, 61-73.

22. Jingqiang Chen, Hai Zhuge (2019). Extractive summarization of documents with images based on multimodal RNN. Future Generation Computer Systems. 99, 186-196.

23. Rajib Ghosh, Chirumavila Vamshi, Prabhat Kumar (2019). RNN based online handwritten word recognition in Devanagari and Bengali scripts using horizontal zoning. Pattern Recognition. 92.203-218.

24. Haiqing Ren, Weiqiang Wang, Chenglin Liu (2019). Recognizing online handwritten Chinese characters using RNNs with new computing architectures. Pattern Recognition. 93, 179-192.

25. Ruoxuan Cui, Manhua Liu, the Alzheimer's Disease Neuroimaging Initiative (2019). RNN-based longitudinal analysis for diagnosis of Alzheimer's disease. Computerized Medical Imaging and Graphics. 73, 1-10

26. Jinyong Wang, Ce Zhang (2018). Software reliability prediction using a deep learning model based on the RNN encoder-decoder. Reliability Engineering \& System Safety. 170, 73-82

27. Routraya, P. Kanungo (2012). Genetic Algorithm Based RNN Structure for Rayleigh Fading MIMO Channel Estimation. Procedia Engineering. 30, 77-84.

28. Chen Wang, Guohua Peng, Bernard De Baets (2020). Deep feature fusion through adaptive discriminative metric learning for scene recognition, Information Fusion, 63, 1-12.

29. Yujuan Ding, Wai Keung Wong, Zhihui Lai, Zheng Zhang (2020). Discriminative dual-stream deep hashing for large-scale image retrieval. Information Processing \& Management. 57(6), 1-15

30. Monica M. Y. Zhang, Kun Shang, Huaming Wu (2019). Deep compact discriminative representation for unconstrained face recognition. Signal Processing: Image Communication. 75, 118-127.

31. Saad Shakeel, Kin-Man Lam (2019). Deep-feature encoding-based discriminative model for age-invariant face recognition. Pattern Recognition, 93, 442-457

32. Haibo Zhang (2018). Geometric discriminative deep features for traffic image analysis. Journal of Visual Communication and Image Representation. 57, 163-171

33. Yan Liu, Shusen Zhou, Qingcai Chen (2011). Discriminative deep belief networks for visual data classification. Pattern Recognition. 44(10),2287-2296

34. Mansoor Iqbal, M. Shujah Islam Sameem, Nuzhat Naqvi, Shamsa Kanwal, Zhongfu Ye (2019). A deep learning approach for face recognition based on angularly discriminative features. Pattern Recognition Letters. 128, 414-419

35. Haijun Liu, Jian Cheng, Wen Wang, Yanzhou Su, Haiwei Bai (2020). Enhancing the discriminative feature learning for visible-thermal cross-modality person re-identification. Neurocomputing. 398, 11-19 
36. Rui Huang, Wei Feng, Mingyuan Fan, Liang Wan, Jizhou Sun (2018). Multiscale blur detection by learning discriminative deep features. Neurocomputing, 285, 154-166.

37. Yisen Liu, Songbin Zhou, Wei Han, Weixin Liu, Chang Li (2019). Convolutional neural network for hyperspectral data analysis and effective wavelengths selection. Analytica Chimica Acta. 1086, 46-54.

38. Abderrahim Mesbah, Aissam Berrahou, Hicham Hammouchi, Hassan Berbia, Mohamed Daoudi (2019). Lip reading with Hahn Convolutional Neural Networks. Image and Vision Computing. 88, 76-83.

39. Jie Shao, Yongsheng Qian (2019). Three convolutional neural network models for facial expression recognition in the wild. Neurocomputing. 355, 82-92.

40. Sérgio F. Chevtchenko, Rafaella F. Vale, Valmir Macario, Filipe R. Cordeiro (2018). A convolutional neural network with feature fusion for real-time hand posture recognition. Applied Soft Computing. 73, 748-766.

41. Måns Larsson, Yuhang Zhang, Fredrik Kahl (2018). Robust abdominal organ segmentation using regional convolutional neural networks. Applied Soft Computing. 70, 465-471.

42. Ima Essiet, Yanxia Sun, Zenghui Wang (2019). Big data analysis for gas sensor using convolutional neural network and ensemble of evolutionary algorithms. Procedia Manufacturing. 35, 629-634.

43. Jonathan Gordon, José Miguel Hernández-Lobato (2020). Combining deep generative and discriminative models for Bayesian semi-supervised learning. Pattern Recognition. 100, 1-13

44. Mourad Gridach (2020). Hybrid deep neural networks for recommender systems. Neurocomputing. 413, 23 30 .

45. Ivano Lauriola, Claudio Gallicchio, Fabio Aiolli (2020). Enhancing deep neural networks via multiple kernel learning. Pattern Recognition. 101, 1-12.

46. Kejun Wang, Xiaoxia Qi, Hongda Liu, Jiakang Song (2018). Deep belief network based k-means cluster approach for short-term wind power forecasting. Energy, 165, 840-852.

47. Mingqi Lv, Wei Xu, Tieming Chen (2019). A hybrid deep convolutional and recurrent neural network for complex activity recognition using multimodal sensors. Neurocomputing. 362, 33-40.

48. Muhammad Alam, Fazeel Abid, Cong Guangpei, L. V. Yunrong (2020). Social media sentiment analysis through parallel dilated convolutional neural network for smart city applications. Computer Communications. 154, 129-137.

49. Charissa Ann Ronao, Sung-Bae Cho (2016). Human activity recognition with smartphone sensors using deep learning neural networks. Expert Systems with Applications. 59, 235-244.

50. Konstantinos Charalampous, Antonios Gasteratos (2014). A tensor-based deep learning framework. Image and Vision Computing. 32(1), 916-929

51. Manoharan, Samuel (2020). Patient Diet Recommendation System Using K Clique and Deep learning Classifiers Journal of Artificial Intelligence. 2(2), 121-130.

52. Muthukumar, Vignesh, N. Bhalaji (2020).MOOCVERSITY-Deep Learning Based Dropout Prediction in MOOCs over Weeks. Journal of Soft Computing Paradigm (JSCP). 2(3), 140-152.

53. Vijayakumar, Vinothkanna (2020).Mellowness Detection of Dragon Fruit Using Deep Learning Strategy. Journal of Innovative Image Processing (JIIP). 2(1),35-43. 